\title{
Video Article \\ ODELAY: A Large-scale Method for Multi-parameter Quantification of Yeast Growth
}

\author{
Thurston Herricks ${ }^{1}$, Fred D. Mast ${ }^{1,2}$, Song $\mathrm{Li}^{1}$, John D. Aitchison ${ }^{1,2}$ \\ ${ }^{1}$ Institute for Systems Biology \\ ${ }^{2}$ Center for Infectious Disease Research
}

Correspondence to: Thurston Herricks at therrick@systemsbiology.org

URL: https://www.jove.com/video/55879

DOI: doi: $10.3791 / 55879$

Keywords: Cellular Biology, Issue 125, Growth rate, lag time, carrying capacity, fitness assessment, yeast, phenotype assay

Date Published: 7/3/2017

Citation: Herricks, T., Mast, F.D., Li, S., Aitchison, J.D. ODELAY: A Large-scale Method for Multi-parameter Quantification of Yeast Growth. J. Vis. Exp. (125), e55879, doi:10.3791/55879 (2017).

\section{Abstract}

Growth phenotypes of microorganisms are a strong indicator of their underlying genetic fitness and can be segregated into 3 growth regimes: lag-phase, log-phase, and stationary-phase. Each growth phase can reveal different aspects of fitness that are related to various environmental and genetic conditions. High-resolution and quantitative measurements of all 3 phases of growth are generally difficult to obtain. Here we present a detailed method to characterize all 3 growth phases on solid media using an assay called One-cell Doubling Evaluation of Living Arrays of Yeast (ODELAY). ODELAY quantifies growth phenotypes of individual cells growing into colonies on solid media using time-lapse microscopy. This method can directly observe population heterogeneity with each growth parameter in genetically identical cells growing into colonies. This population heterogeneity offers a unique perspective for understanding genetic and epigenetic regulation, and responses to genetic and environmental perturbations. While the ODELAY method is demonstrated using yeast, it can be utilized on any colony forming microorganism that is visible by bright field microscopy.

\section{Video Link}

The video component of this article can be found at https://www.jove.com/video/55879/

\section{Introduction}

Growth phenotypes of microorganisms are a strong indicator of their underlying genetic fitness to a given environmental condition. Growth is classically segregated into 3 different growth regimes: lag-phase, log-phase, and stationary-phase growth ${ }^{1}$. Each growth phase can reveal different aspects of fitness that are dependent on various environmental and genetic conditions. For example, lag time, or the length of time an organism spends in lag-phase before the start of exponential growth, can be indicative of an organism's ability to respond to altered environmental conditions ${ }^{2}$. Doubling time during log-phase growth, the most common metric of cellular fitness, reveals the overall efficiency of an organism's ability to divide by metabolizing and utilizing environmental materials for replication. Stationary phase, where growth after log-phase is rapidly reduced, is another indicator of fitness, which is regularly used as a growth endpoint in spot-based yeast growth assays.

Several yeast growth assays are currently available and considered standard methods for evaluating growth phenotypes in yeast ${ }^{3,4,5}$. These assays are primarily based on methods for growing yeast either on solid or in liquid media. On solid media, colony pinning assays transfer a small number of cells onto solid agar with a pin, and yeast cells are allowed to grow for a defined period of time. Colonies are then imaged and their sizes are compared at a terminal endpoint ${ }^{6}$. These colony pinning assays have proven robust and scalable to generate genome-wide screens. More recently, periodic imaging using flat-bed scanners and Single Lens Reflex (SLR) cameras have been incorporated into these assays to record colony growth over time ${ }^{7,8,9}$. However, the resolution of these devices prevents them from detecting single cells and thus, these colony pinning assays do not directly observe lag time and cannot observe variation between the individual cells that grow into colonies.

Liquid-based growth assays have also been employed to perform genome-wide screens ${ }^{3}$. Coupling a liquid growth assay with time-lapse microscopy revealed population heterogeneity in the doubling time of genetically identical individual cells, which offers an important perspective for understanding genetic regulation and environmental adaptation. However, this assay does not measure other aspects of growth such as lag time and carrying capacity ${ }^{10}$. Here we present a method to characterize all three growth phases of colony forming microorganisms on solid media using an assay we term ODELAY ${ }^{11}$. ODELAY consists of utilizing high throughput time-lapse microscopy to record images of single cells growing into colonies on solid media. This population of individual cells growing into colonies reveals the underlying population heterogeneity, which is not detected by other less sensitive measurements such as terminal endpoint scoring. We demonstrate the method on yeast, but ODELAY may be applied to any organism that shows contrast in bright field microscopy. 


\section{Preparation of Agarose Gel Stock}

1. Weigh out $2 \mathrm{~g}$ of high purity agarose.

2. Add the agarose to a $500 \mathrm{~mL}$ bottle and record their combined mass.

3. Calculate the target mass of the bottle plus $2 \mathrm{~g}$ agarose with $150 \mathrm{~g}$ of ultra-pure water, then add $150 \mathrm{~g}$ of ultra-pure water to within $0.1 \mathrm{~g}$ of this target mass.

4. Note the mass of the bottle plus the agarose and water.

5. Place the bottle in a microwave and make sure to fit the bottle with a loose cap on top to minimize water evaporation while heating the agarose.

6. Microwave the bottle in $15-20 \mathrm{~s}$ bursts followed by briefly swirling the bottle to mix the agarose and water. Repeat this procedure until the solution is boiling and the mixture is homogeneous.

CAUTION: Take care to avoid scalding skin with steam escaping from the bottle. Also, the bottle will become hot to the touch and appropriate protection, such as an autoclave glove, is required to avoid injury.

7. After the molten agarose is homogeneous, weigh the bottle again and add ultrapure water to within $0.1 \mathrm{~g}$ of the original mass. This will replace any water lost due to evaporation.

8. Before the molten agarose solidifies, swirl to mix in the added water to ensure homogeneity.

9. Aliquot $15.2 \mathrm{~g}$ of agarose into $9-50 \mathrm{~mL}$ plastic tubes.

10. Refrigerate the tubes until needed.

\section{Preparing ODELAY Agarose Media}

1. Heat $400 \mathrm{~mL}$ of deionized water to boil in a covered beaker, on a hotplate under gentle stirring with a magnetic stir bar.

2. Add $2 \mathrm{~mL}$ of $10 \mathrm{X}$ media (e.g., Yeast Extract Peptone (YEP), or Complete Supplement Mixture (CSM)) to a $15.2 \mathrm{~g}$ agarose aliquot in a $50 \mathrm{~mL}$ conical tube from step 1.10 .

NOTE: CSM medium tends to stick to the glass slides. If using CSM media formulations, add $5 \mu \mathrm{L}$ of $50 \%$ wt Polyethylene glycol (PEG) in sterile water to the medium formulation. The PEG prevents the agar from sticking to the glass slides during mold release and does not inhibit yeast growth.

3. Add additional $100 \mathrm{X}$ nutrient supplements, if needed, and use water to bring the total added volume from this step to $1 \mathrm{~mL}$.

4. Weigh the agarose aliquot with added media and supplements before placing the $50 \mathrm{~mL}$ conical tube into boiling water from step 2.1 .

5. After $16 \mathrm{~min}$, take out the $50 \mathrm{~mL}$ tube and homogenize the mixture using a vortex. Keep the cap fastened tight on the $50 \mathrm{~mL}$ conical tube. NOTE: A lid on the beaker helps heat the whole tube evenly otherwise a film of solid agarose will form and possibly not melt. Also during this time, it is convenient to assemble the agarose mold (Figure 1).

6. Homogenize the mixture using a vortex.

7. Boil for an additional 2 min to ensure all agar is mixed and melted.

8. Weigh tube and replace any mass lost with ultra-pure sterile water

9. Add $2 \mathrm{~mL}$ of $10 \mathrm{X}$ carbon-source reagent (e.g., $20 \% \mathrm{w} / \mathrm{v}$ glucose) and vortex to obtain agarose medium solution. NOTE: Separation of Glass Slides: When assembling the mold, care must be taken to make sure the long spacers are placed in the mold with the correct orientation. This is because as the laser cuts the acrylic, it tends to cut as a cone leaving the part with slightly angled sides instead of exactly $90^{\circ}$ sides. Then when the mold is placed on the benchtop to release the glass from the agar, the edge of spacer should be at an acute angle with the agar. The acute angle helps compress the edge of the agar away from the upper piece of glass as the outside edge of the mold spacer is pivoted about the lower edge (see Figure 1). The result is a more consistent separation of the agar from the top piece of glass.

NOTE: Mold release agents applied to the glass like oils, silicon sprays or even commercial window treatments should not be used because they will contaminate the surface of the agar and possibly inhibit growth. These methods take some practice to be consistent.

10. Clean four 2 in $\times 3$ in $\times 1 \mathrm{~mm}$ thick glass slides with $70 \%$ ethanol and dry using forced air.

11. Clean acrylic molds with $70 \%$ ethanol, dry, and assemble as shown in Figure 1. Take the three bottom pieces, as shown, and assemble them so that the two identical pieces sandwich the third piece (Figure 1B). Clamp the base pieces on each side using two small binder clips (Figure 1C). Place the uprights into the mold making sure that the laser kerf is correctly positioned (Figure 1E).

12. Place the cleaned and dried glass slide on the mold and hold it in place while placing a second glass slide on the other side. Clamp the assembly with a larger binder clip (Figure 1D). Clamp down both slides with larger binder clips (Figure 1D). Make sure the upper binder clip is in contact with the glass slide overlapping with the acrylic.

13. Add remaining binder clips. Again, make sure the clamps contact the slide where it overlaps the acrylic (Figure 1D). NOTE: Before filling the assembled mold with molten agar, ensure the edges are sealed by pipetting about $70 \mu \mathrm{L}$ of molten agar media along the inner side of the mold. This agar will quickly solidify and prevent any leaks.

14. Fill the mold with molten agar, making sure to avoid trapping air bubbles in the mold. NOTE: This can be accomplished by slowly pipetting the molten agar along the edge of the mold. After it is filled, allow the mold to cool for 40 $\min$ to $1 \mathrm{~h}$ at about $23^{\circ} \mathrm{C}$ ambient temperature. If allowed to cool too long the agar may fracture in the mold.

NOTE: Mold Separation: Proper removal of the mold from the cast agar is essential to ensuring a uniform solid agar pad for yeast growth. Failure to ensure efficient separation of the mold at this step will cause growth differences that are attributable to imperfections in the solid agar pad. Practicing this step a few times is recommended.

15. Start by removing the bottom binder clip. Remove the bottom part of the mold that consists of the three sandwiched pieces. Hold the slides by compressing them and then remove the binder clips. Be sure not to bump the mold sides while removing the binder clips. This will deform the media. 
16. Place the mold on the edge of the bench top so that the side of the mold with the blue dot is facing up and in the lower left corner (Figure 1E). Place thumbs underneath the acrylic and the first finger on the top towards the inner edge of the mold.

17. Slowly and carefully push up with thumb against the mold, as if pivoting the mold spacer about its lower edge (Figure 1F). Apply constant but slowly increasing pressure. Users will see a break and air bubble appear along the line where the top glass covers the mold spacer.

18. After seeing the initial break line, continue pushing up with thumbs and apply constant pressure. The agar should start breaking away from the glass at this point (Figure 1F). Continue to pivot the mold upwards. This will lift the glass without sliding it. A line where the agar is peeling away from the glass should continue to move away from the initial break line.

NOTE: At this point, the agar is sticking to both the bottom and top of the glass. This will occasionally occur with the left most edge. As long as the area deformed is not in the area where yeast is spotted, this should be fine.

19. As the glass comes completely free, grab it and remove it completely from the mold. Then remove the other mold piece using a similar motion to lift the piece free without moving the agar. Place the slides in a sterile tip box with some sterile high purity water in the bottom. Close the box and store at $4{ }^{\circ} \mathrm{C} \mathrm{O} / \mathrm{N}$ for use the next day. If stored for longer, the growth rates will become inconsistent.

\section{ODELAY Culture Preparation}

1. Lay out strains in a 96-well plate for $\mathrm{O} / \mathrm{N}$ growth.

2. The next day, dilute $20 \mu \mathrm{L}$ of overnight culture into $200 \mu \mathrm{L}$ media for $220 \mu \mathrm{L}$ total volume in a new plate.

3. Measure the optical density at $600 \mathrm{~nm}(\mathrm{OD} 600)$ of each culture in a plate reader. Using a plate reader and a liquid handling robot, follow the automated dilution protocol of steps 3.3.1 - 3.3.6. Manually dilute the cultures in the plate to approximately 0.1 OD600 (optional).

1. On the plate reader, hit "Experiment" under "create new". Choose ODELAYDilution.exp and run the experiment. Enter experiment name as ODELAY "Date" "Time" "Experiment Name" iteration.

2. Click on the statistics tab and then click on the Excel icon. Save the data to a thumb drive and transfer it to a liquid handling robot computer.

3. Open the Robot's Layout and Method Editor. Open the Method file "ODELAYDilution_v1.med". Run the Executable "Convert_SynergyFiles.exe". Enter 0.09 for target OD600.

4. Click on "Glu Correction" and "Gal Correction" to 0.05. Measure blank media in an identical plate. Click on "Generate File". Select the file in the method editor. Click on the stoplight icon to start the method to open the runtime dilution program.

5. Make sure tubes are uncapped and plates have lids removed and dust covers are removed from tips. Load plates, tubes and tips onto the liquid handling robot's deck, as indicated by the deck layout.

6. Click the "Play" button to run the dilution program. Select the correct number of $50 \mu \mathrm{L}$ tips. Select the correct number of $300 \mu \mathrm{L}$ tips. Wait about 12 min for the process to run.

4. Take the dilution plate from the robot, cover the tips, and recap the $15 \mathrm{~mL}$ media tubes. Culture the plate for $5-6 \mathrm{~h}$ at $30{ }^{\circ} \mathrm{C}$.

5. About $1-2 \mathrm{~h}$ before starting the second dilution make sure to turn on the incubation chambers for the microscope. Allow them to equilibrate at ${ }^{\circ} \mathrm{C}$ or the temperature required for the experiment.

6. Repeat the dilution steps $3.3-3.4$, but dilute cultures to an OD600 of $0.01-0.02$ for spotting the cultures on agarose slides.

7. Cover the dilution plate with a metal freezer seal.

8. Sonicate the dilution plate in an ice bath for $30 \mathrm{~s}$ with the plate floating in ice water using a centrifuge metal bucket-fitting to hold the plate (Figure 2A). Transfer the sonicated cultures from the dilution plate to a flat bottom plate. Label $4 \times 96$-well flat bottom plates $1,2,3$ and 4 (Figure 2B).

1. Transfer $150 \mu \mathrm{L}$ of the dilution plate from wells A01 - D06 into wells C04 - F09 of plate labeled 1. Then transfer $150 \mu \mathrm{L}$ of the dilution plate from wells A07 - D12 into wells C04 - F09 of plate labeled 2. Then transfer $150 \mu \mathrm{L}$ of the dilution plate from wells E01 - H06 into wells C04 - F09 of plate labeled 3. Finally transfer $150 \mu \mathrm{L}$ of the dilution plate from wells E07 - H12 into wells C04 - F09 of plate labeled 4.

9. Move on to step 4, Spotting on Agar.

\section{Spotting on Agar using an Automated Liquid Spotting Robot}

1. Remove the agarose slides stored at $4{ }^{\circ} \mathrm{C} \mathrm{O} / \mathrm{N}$ (from step 2.19) from their humidified sterile pipette boxes.

2. If needed, trim the corners of the agarose medium with a clean razor blade to ensure that they will fit on the slide chamber.

3. Remove the slide clamp off the mount. Carefully place the agar plate into the recessed area of the stage chamber mount. NOTE: Make sure the orientation of the slide is consistent from experiment to experiment.

4. Check to make sure the clamp is flush with the bottom of the chamber. If it is crooked, then the slide may not be fully seated in the recessed area. Place the leveling spacer into the center plate position of the spotting robot. This spacer provides contact for the leveling screws so that the slide chamber can be leveled with the tips.

5. Place the plates on the table in order of their quadrant. Plates 1, 2, 3 and 4 ordered from left to right (Figure 2B).

6. Lay out the tips so that the inner 24 wells C04 - F09 are occupied with tips into 4 empty tip boxes. A fifth box will need to have one tip in the C10 position, as well as 4 tips with their ends cut off in the A01, A12, H01, and H12 positions. These 4 cut off tips provide stability for the tip plate clamp (Figure 2C and 2D).

7. Remove the top cover from the slide chamber mount.

8. Place the first tip punch into the start site of the robot control spotting program. This should punch a hole in the agarose which will later be used for aligning the origin coordinate. Place plate 1 on the liquid handling robot to spot the first quadrant. Make sure the lid is removed and continue the spotting program.

9. Check to make sure all spots are present. Also, wait $\sim 30 \mathrm{~s}$ for them to dry. When the spots are about 1 mm in diameter, the program may continue. Empty the used tips into the biohazard container, and place fresh tips on the robot. Swap out plate 1 for the $2^{\text {nd }}$ quadrant plate.

10. Repeat for the third quadrant. And repeat again for the $4^{\text {th }}$ quadrant.

11. When the spots are dry, replace the slide chamber cover and flip the apparatus over.

12. Install the tubing connections with an air filter. 
13. Place a glass slide on the top side of the chamber.

NOTE: This slide is crucial for reducing the heat flux to the agar and minimizes the formation of condensation onto the objective cover slip side of the chamber. Do not forget this cover slip. Depending on the microscope configuration, this cover slip prevents heating from the illumination side from causing condensation on the objective side.

14. Position a fan to blow hot air onto the objective side of the chamber. This fan prevents condensation from forming on the cover slip. Set the air-flow rate through the bubbler to $10 \mathrm{~mL} / \mathrm{min}$.

\section{Running ODELAY on Microscope}

1. Run the script "ODELAY_Microscope_Control.m".

2. Click on the "Shutter" to open the transmitted light shutter and then the "Focus" button to initiate the high camera rate (Figure 3A, Red Arrows).

3. Click on "Go Origin" and move the stage to find the origin mark that was punched in the agar.

4. Then move slightly to the right to focus on yeast cells spotted in area E07.

5. Move back to the origin punch and center it in the field of view.

6. Set the origin to this value by pressing the "set" button (Figure 3A, Blue Arrows). Now move to position $\mathrm{H} 18$ and focus using the hex screw closest to that location. Then move to position L07 and focus using the hex screw closest to that location. Then move to position E07 and focus using the hex screw closest to that location.

7. Repeat steps $5.5-5.7$ as necessary until those positions remain in focus.

8. Check the focus in the center and at the edges at spots E12, H12, L12. Adjust the autofocus range if the focus Z-values, as indicated by the $Z$-value, are greater than \pm the autofocus range (e.g., set the autofocus range to $60 \mu \mathrm{m}$ the Z-value for a center spot that is greater than \pm 40 $\mu \mathrm{m})$.

9. Press the reset button as this will activate the button properties, and then press ODELAY (Figure $\mathbf{3 A}$, Green Arrows). Choose the directory to save the data. Make sure that enough drive space is available. NOTE: The microscope collects data for $48 \mathrm{~h}$, or until the program is closed.

\section{Processing ODELAY Data}

1. Open the ODELAY_IPT.exe or use the script ODELAY_Image_Processing_Tool_v7.m script (Figure 3C).

2. Prepare an *ODELAYYExpDisc.xIsx excel spreadsheet.

3. Name the experiment in cell B1.

4. Select the Image Directory where the Image files E07 - H18 are stored.

5. Select the Data Directory where data will be written.

6. Write the date when the experiment was started into cell B04. Use the format MM/DD/YYYY.

7. Write the dilution time in cell $\mathrm{CO4}$. This time is roughly five min before the first image is collected. Use the format $\mathrm{HH}: \mathrm{MMpm}$ where $\mathrm{HH}$ is for $\mathrm{h}$ and $\mathrm{MM}$ is for $\min$.

8. Add in the strain names according to the source plate (step 3.1). Due to the way strains are spotted, they are rearranged on the ODELAY agarose slide. Cells in the spreadsheet B31-B126 are the source plate, while cells C31 - C126 are the spot locations in the ODELAY agar plate.

9. Then press the "Process Data" button and select the *ODELAYExpDisc.xlsx that was just prepared.

10. Wait 16-24 $\mathrm{h}$ depending on the computer system used to process data.

11. When the images are processed, a directory named "ODELAY Well Data" and a file "*_Index_ODELAYData.mat" will appear. Press the "Load Data" button and select the "*_Index_ODELAYData.mat" file that was just generated. This will load the data set that was just processed. The "*" in the file name will be listed according to the experiment name entered into the excel spreadsheet.

12. After loading the data, inspect it using the time bar found across the bottom, click on an image square to see growth curves for that spot, or find a well of interest in the list on the left and then load the images for that list.

13. Generate histograms of population growth parameters by pressing the "Violin Plot" button. This will generate a plot shown in Figure 4 or Figure 6.

\section{Representative Results}

Example images of yeast growing in time-lapse microscopy are shown in Figure 3B. After processing the time-lapse images, a representative data set comparing yeast strains BY4741 \& BY4742 is shown in Figure 4. In this example dataset, there is very little variation in doubling time between different positions on the plate. If the agarose medium is prepared poorly, then an obvious deviation in both doubling time and lag time would be apparent in the spot positions that coincide with the deformed region of the agarose gel. While doubling times appear to be relatively uniform, this example shows variations in lag time measurements. A more consistent data set is shown in Figure 6. In this dataset both lag time and doubling time are uniform. 


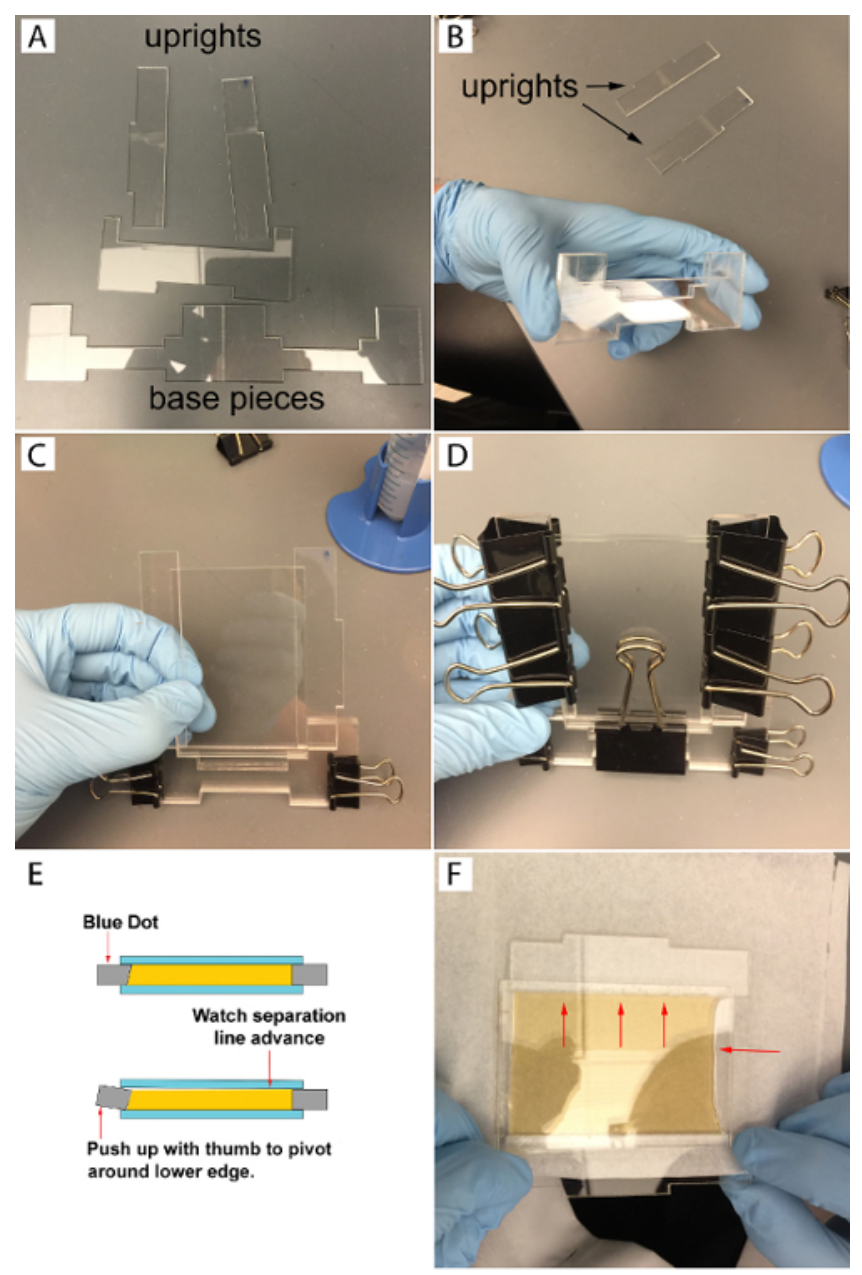

Figure 1: Agar Mold Assembly.

The components of the agar mold are shown in (A). Assemble the base as shown in (B) and then clamp the base with small binder clips. Place the longer upright pieces in the cavity of the base (C) and then camp the slides to the mold as shown in (D). A side view showing the angle of the mold and orientation of the mold kerf needed for consistent separation of the agar from the glass slide (E). Note the position of the thumb and forefingers, as well as the straight line of the agar separating from the slide $(\mathbf{F})$ and note the horizontal arrow. The separation line should move evenly away in the direction of the vertical arrows. Please click here to view a larger version of this figure. 


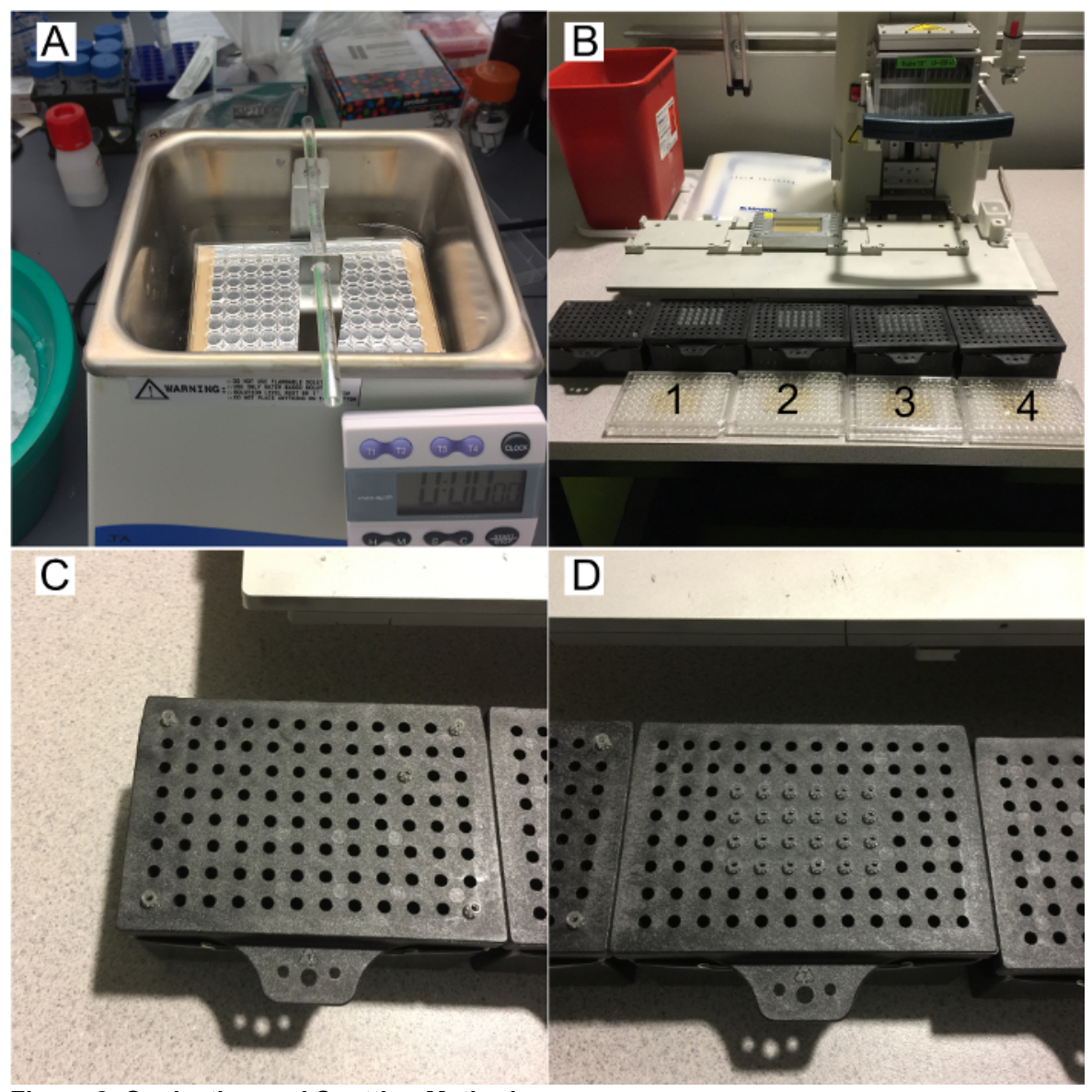

Figure 2: Sonication and Spotting Method.

Sonicate the plate in ice water and using a centrifuge bucket holder to help support the plate (A). Lay the plates from steps 3.8 .1 out for the spotting onto the agarose plate (B). Also, arrange the tips so that the left most tip box has one tip at position C10 and then four other tips with their ends cut off so they do not crash the plate holder (C). Place the remaining tips in four boxes so that the inner 24 tips positions are occupied (D). Please click here to view a larger version of this figure. 

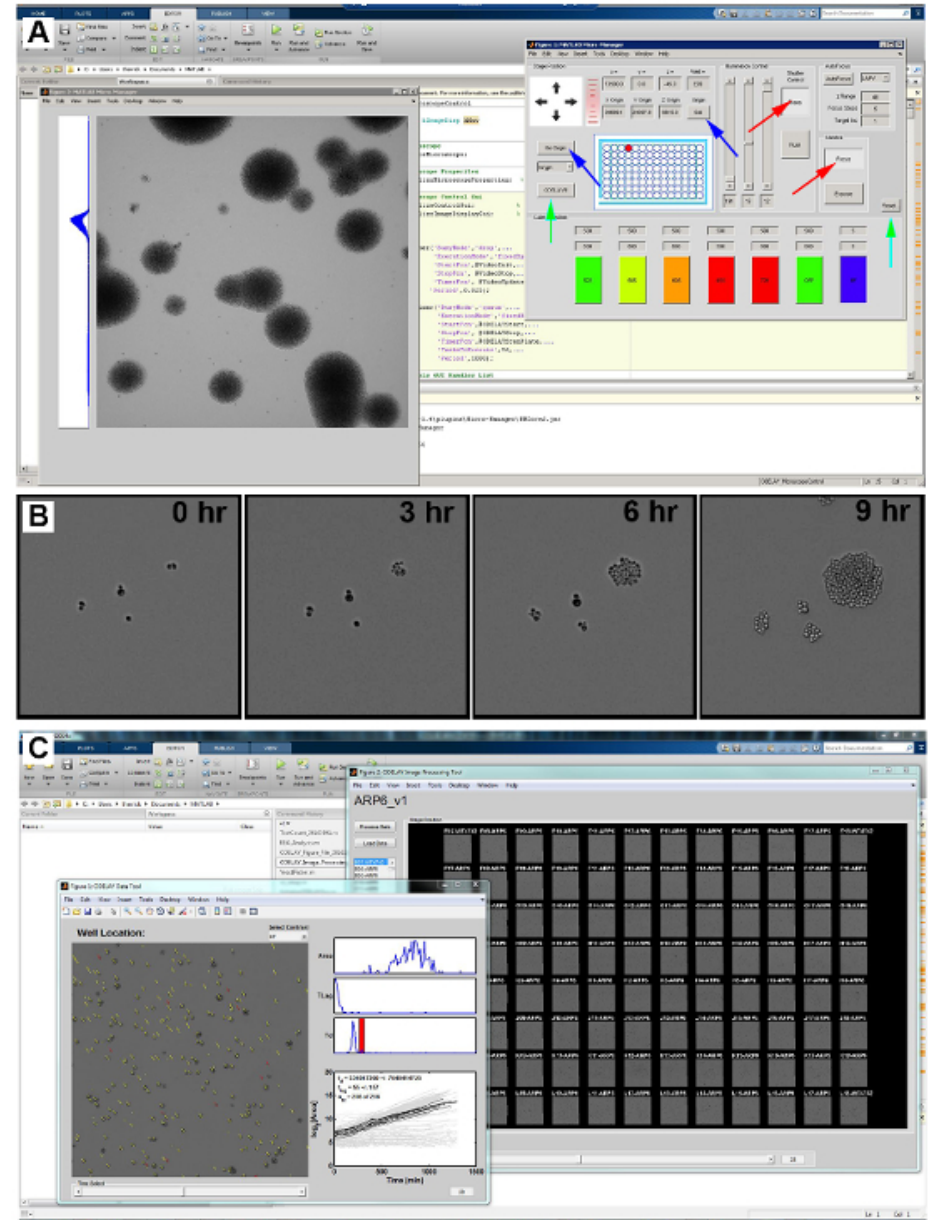

Figure 3: ODELAY Graphical User Interface.

A screen shot of the Graphical User Interface for "ODELAY_Microscopecontrol.m" (A). This interface allows monitoring of the camera and for adjusting the microscope illumination settings for epifluorescent and bright-field modes. Red arrows point to the Focus and Transmitted buttons that activate the camera to acquire images quickly and open the transmitted light shutter respectively. Blue arrows are used for moving the stage for the origin and then setting the origin with the "Go Origin" button and "Set" button. Green arrows point to "Reset" and "ODELAY!!!" buttons that reset ODELAY image modes to the current conditions and initiates ODELAY image collection. Time-lapse images of yeast growing on solid medium at $0,3,6$, and $9 \mathrm{~h}$ after spotting (B). A screen shot of the Graphical User Interface for "ODELAY_IPT.m" or the ODELAY Image Processing Tool $(\mathbf{C})$. Please click here to view a larger version of this figure. 


\section{BY4741-42 Eval v2}

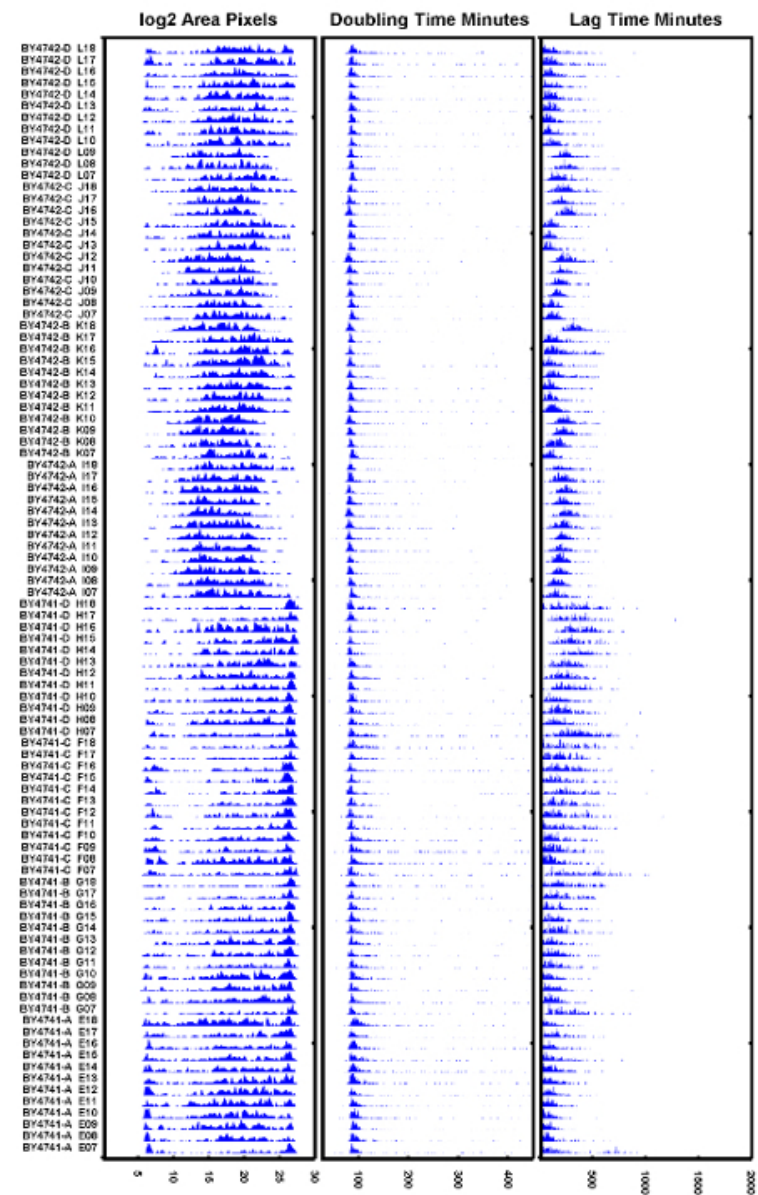

Figure 4: Example ODELAY Output.

This dataset compares strains BY4741 and BY4742 on YPD media. This figure is an example of a well-prepared agarose slide; however, autofocus settings are not optimal. The data presented in each column, from left to right, are: the carrying capacity in Log ${ }_{2}$ of the colony area; doubling time, given in min; and lag time, given in min. In this example, doubling times of all spots on the agar slide line up well with a small amount of increased doubling time towards the column. However, lag times vary considerably in this dataset. Please click here to view a larger version of this figure. 

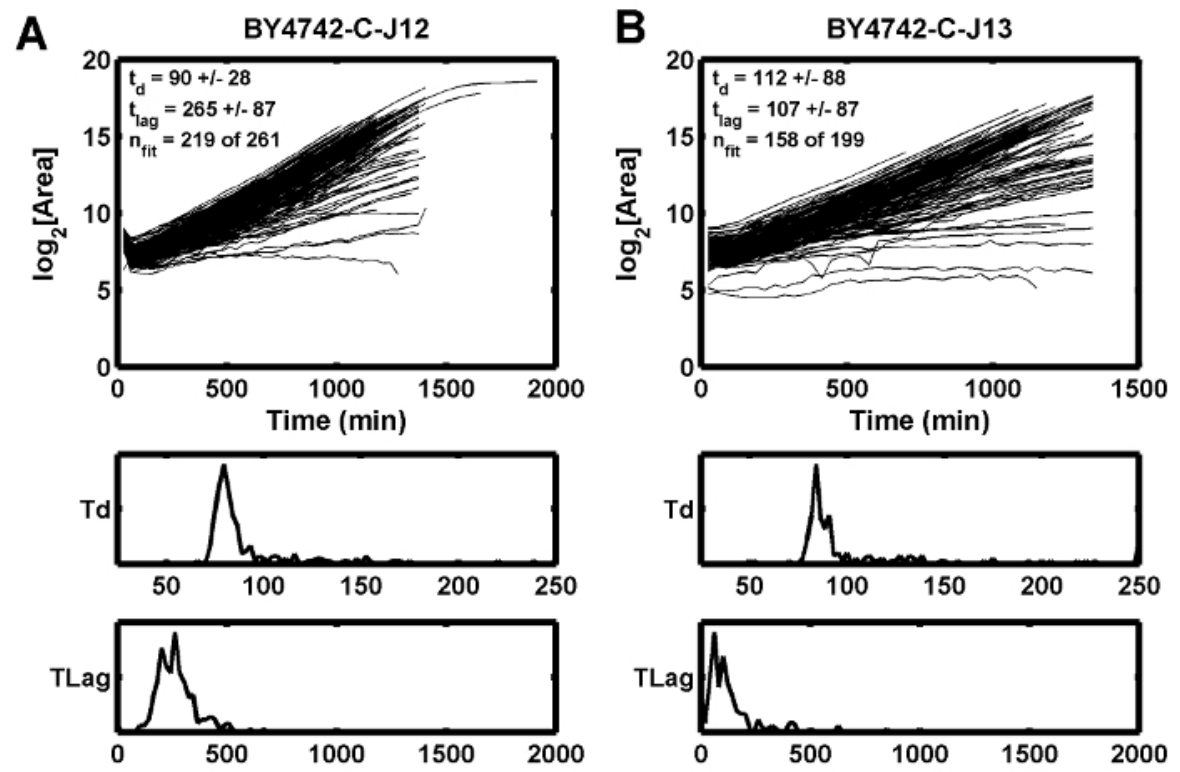

Figure 5: Growth Curve Examples.

This example demonstrates how poor initial focus can cause the estimated lag time $\left(t_{\text {lag }}\right)$ to increase $(\mathbf{A})$, while the adjacent position shows a shorter lag time (B). $t_{d}$ is the doubling time in $\min$, and $t_{\text {lag }}$ is the lag time in min. Please click here to view a larger version of this figure.

\section{BY4742 Diode v2}

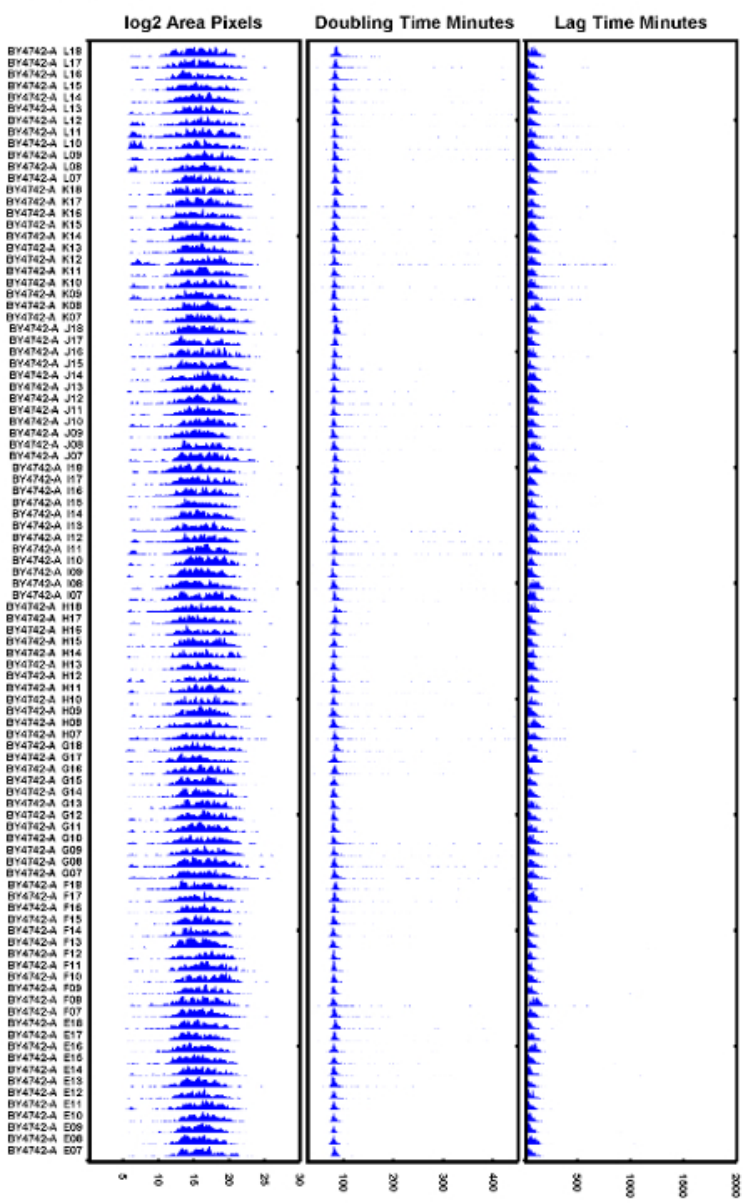

Figure 6: Example of Well Executed Test Experiment.

An example of the BY4742 strain tested after replacing a tungsten halogen bulb with a diode illuminator and ensuring the autofocus is set correctly. All doubling times appear to overlap well and the lag times appear to be consistent. Please click here to view a larger version of this figure. 


\section{Discussion}

The ODELAY assay has several critical points for ensuring reproducible and reliable phenotypic measurements. The first critical point is consistent preparation of the yeast cultures. Care must be taken to harvest the yeast cells from logarithmic growth. If the cultures have saturated, then their population heterogeneity will be increased which may obfuscate heterogeneity caused by genetic or environmental (e.g., carbon source) factors ${ }^{11}$. The second critical point is consistent preparation of the media. In general, a large volume of $10 \mathrm{X}$ stock media solution should be generated and then used over time to minimize batch effects. Formulating media by weight, whenever possible, helps improve the consistency of the medium over time by ensuring the density of agar and the overall water content of the agarose can be closely monitored. The third critical point involves minimizing or eliminating any mechanical deformation of the agarose media. Mechanical deformation of the media will most commonly occur during separation of the agarose from the glass slides. As with many laboratory techniques, practice is required to master this step.

Variation in lag time as depicted in Figure 4, is often related to one of the three factors: mechanical deformation of the agarose medium, variation in the molded agar thickness, or an unstable light source. If the agarose medium varies in Z-height across the spotted array, the height variation may overwhelm the range of the autofocus routine, causing the initial images to be slightly out of focus. For this reason, check the focus height at multiple spots in the center and along the edges of the spotted array to ensure the autofocus routine has sufficient Z-range to find focus. If needed, use the Autofocus panel to increase the focus range and increase the number of focusing steps.

A third possible condition that may lead to poor focus is an unstable or flickering light source, which can disrupt the calculated focus score for a specific Z-height. Tungsten halogen bulbs tend to flicker well before the bulbs burn out. The effect of poor focus is observed in one example where the growth curves dip between the first and second time points (Figure 5A), while the adjacent spot does not have the same dip (Figure 5B). In this case, the poor focus condition was alleviated by replacing the tungsten halogen light source.

In practice, the authors have found that to reduce the flicker of $100 \mathrm{~W}$ tungsten halogen bulbs, the bulbs need to be replaced every $500 \mathrm{~h}$ or roughly every 2 months when the microscopes are under heavy use. To avoid poor focus issues from a flickering bulb, replace the tungsten halogen light source often or replace the halogen bulb with a diode light source. An example of a dataset that shows low variation in doubling times as well as more uniform lag times is shown in Figure 6. This dataset was taken with a diode illuminator which provides a more stable illumination over time while performing the autofocus.

While many of the points mentioned here for optimizing media preparation may appear to be obvious, in the literature most large scale screens do not replicate well with each other ${ }^{8,11}$. Therefore, we have carefully described the preparation of cultures and agarose media so that more reproducible phenotypic screens may be generated.

The ODELAY assay is currently limited in throughput when compared to pinning based assays such as synthetic genetic arrays or the ScanO-Matic assay. While these methods increase the number of strains that are measured, they lack an ability to resolve individual cells and thus cannot measure population heterogeneity that we observe within clonal yeast strains. The origin of this population heterogeneity is not currently understood, but the merging of technology and computation as demonstrated here offers an opportunity for objectively addressing the underlying cellular mechanisms ${ }^{12}$.

The authors wish to note that ODELAY is currently only optimized for a specific microscope brand and body type. Modifying ODELAY for other microscope systems is straight forward but will require knowledge of the open source API ${ }^{13}$. However, both the API as well as the ODELAY scripts are written to be easily adapted to different systems and experimental assays.

While ODELAY was originally developed for yeast, we have been able to utilize it without modification to observe growth of Mycobacterium smegmatis. Observation of the other colony forming microorganisms is possible with alterations to the source code provided ${ }^{11}$. In general, ODELAY is a powerful and flexible tool for comparing microorganisms grown under different environmental conditions and genetic perturbations.

\section{Disclosures}

The authors have nothing to disclose.

\section{Acknowledgements}

The authors acknowledge support for this work by grants U54 RR022220 and P50 GM076547 to J.D.A from the U.S. National Institutes of Health. F.D.M. is a postdoctoral fellow with the Canadian Institutes for Health Research. We also thank the Luxembourg Centre for Systems Biomedicine and the University of Luxembourg for support.

\section{References}

1. Zwietering, M. H., Jongenburger, I., Rombouts, F. M., van't Riet, K. Modeling of the bacterial growth curve. Appl Environ Microbiol. 56 (6), 1875-1881 (1990).

2. Sellick, C. A., Campbell, R. N., Reece, R. J. Galactose metabolism in yeast-structure and regulation of the leloir pathway enzymes and the genes encoding them. Int Rev Cell Mol Biol. 269, 111-150 (2008).

3. Yoshikawa, K., et al. Comprehensive phenotypic analysis for identification of genes affecting growth under ethanol stress in Saccharomyces cerevisiae. FEMS Yeast Res. 9 (1), 32-44 (2009). 
4. Bryan, A. K., Goranov, A., Amon, A., Manalis, S. R. Measurement of mass, density, and volume during the cell cycle of yeast. Proc Natl Acad Sci U S A. 107 (3), 999-1004 (2010).

5. Baryshnikova, A., et al. Quantitative analysis of fitness and genetic interactions in yeast on a genome scale. Nat Methods. 7 (12), 1017-1024 (2010).

6. Costanzo, M., et al. The genetic landscape of a cell. Science. 327 (5964), 425-431 (2010).

7. Collins, S. R., et al. Functional dissection of protein complexes involved in yeast chromosome biology using a genetic interaction map. Nature. 446 (7137), 806-810 (2007).

8. Zackrisson, M., et al. Scan-o-matic: High-Resolution Microbial Phenomics at a Massive Scale. G3 (Bethesda). 6 (9), $3003-3014$ (2016).

9. Bean, G. J., Jaeger, P. A., Bahr, S., Ideker, T. Development of ultra-high-density screening tools for microbial 'omics'. PloS One. 9 (1), e85177 (2014).

10. Levy, S. F., Ziv, N., Siegal, M. L. Bet hedging in yeast by heterogeneous, age-correlated expression of a stress protectant. PLoS Biol. 10 (5), e1001325 (2012).

11. Herricks, T., et al. One-Cell Doubling Evaluation by Living Arrays of Yeast, ODELAY! G3 (Bethesda). 7 (1), $279-288$ (2017).

12. Mast, F. D., Ratushny, A. V., Aitchison, J. D. Systems cell biology. J Cell Biol. 206 (6), 695-706 (2014).

13. Edelstein, A. D., et al. Advanced methods of microscope control using $\mu$ Manager software. J Biol Methods. 1 (2), e10 (2014). 